\title{
Persistence of a Reduced-collagen-producing Phenotype in Cultured Scleroderma Fibroblasts after Short-term Exposure to Interferons
}

\author{
Matthew R. Duncan and Brian Berman \\ Dermatology Service, Veterans Administration Medical Center, Martinez, California 94553; and Department of Dermatology, University \\ of California, Davis, School of Medicine, Davis, California 95616
}

\begin{abstract}
Transient exposure to inflammation-associated, fibroblast-stimulatory factors appears to initiate fibrosis by inducing the persistently activated phenotypes displayed by fibroblast cultures derived from scleroderma skin and other fibrotic tissues. To determine whether one class of fibroblast-inhibitory factors, the interferons (IFNs), plays a role in terminating fibrosis by acting as persistent fibroblast deactivators, we inhibited (40-60\%) the growth and collagen production of normal dermal fibroblasts and hypercollagen-producing scleroderma fibroblasts by shortterm exposure to IFN- $\alpha, \beta$, or $\boldsymbol{\gamma}$. During subsequent subculture in the absence of IFNs, the growth and collagen production of normal fibroblasts and the growth of scleroderma fibroblasts increased to untreated control levels after two to three passages. In contrast, collagen production by scleroderma fibroblasts remained inhibited for at least five passages (18 cell doublings) and was not further suppressed by subsequent IFN exposure. These data suggest that IFNs may help terminate fibrosis by suppressing persistently activated fibroblast functions.
\end{abstract}

\section{Introduction}

Fibroblasts are responsible for the production and maintenance of the connective-tissue matrix. Under basal conditions, the growth and synthetic activities of resident fibroblasts are of a limited nature and likely controlled only by interaction with native connective-tissue matrix components $(1,2)$. However, after either traumatic or pathological tissue injury, resident fibroblasts undergo a metabolic activation and exhibit the increased growth and synthetic rates required to effect the fibrotic repair of injured tissue. This fibroblast activation is believed to be controlled by the presence of numerous stimulatory factors released into the tissue fluid environment as a result of blood vessel disruption and the infiltration and activation of platelets, neutrophils, monocytes/macrophages, and T lymphocytes during the inflammation and granulation tissue phases of the fibrotic response (3-16).

Whereas fibrosis in response to traumatic injury is usually a transient process resulting in healed wounds with minimal scar formation, pathological fibrosis is often a persistent chronic process resulting in excessive tissue scarring. Cultured fibroblasts

This study was presented in preliminary form at the national meeting of the Society for Investigative Dermatology, 1-4 May 1986, Washington, DC, and has been published in abstract form (1986. J. Invest. Dermatol. 86:473; and 1986. Clin.Res. 34:747A).

Address correspondence to Dr. Duncan, Dermatology Service (190), VA Medical Center, 150 Muir Road, Martinez, CA 94553.

Received for publication 11 August 1986 and in revised form 16 January 1987.

The Journal of Clinical Investigation, Inc.

Volume 79, May 1987, 1318-1324 derived from sites of pathological fibrosis, such as rheumatoid synovium, and keloid or scleroderma skin, display activated phenotypes characterized by increased production of the connective-tissue matrix components: collagens (17-23), glycosaminoglycans $(17,24)$, and fibronectin $(21)$, as well as altered growth $(17)$ and collagenase $(18,25)$ and prostaglandin $E_{2}$ production (25). Moreover, a portion of these cultured fibroblasts, activated by the in vivo environment from which they were excised, appear to be permanently, or at least persistently, activated as their hyperactive phenotypes persist through numerous subcultures in vitro $(17-20,22-25)$.

Studies investigating the environmental stimulatory factors responsible for inducing pathologic fibroblast activation have recently focused on those produced by mononuclear cells, because it has long been recognized that both $T$ lymphocytes and monocytes/macrophages are present during the inflammatory phase of pathological fibrosis (26-29). These studies indicate that both $\mathrm{T}$ lymphocyte-derived lymphokines $(15,16,29,30)$ and monocyte/macrophage-derived monokines (12-14, 30-33) can stimulate normal fibroblast functions in vitro. Further, in two instances, namely, glycosaminoglycan and prostaglandin $\mathrm{E}_{2}$ production, it has been demonstrated that the stimulated phenotypes induced by culture in lymphokine/monokine supernatants are retained in the absence of the stimulating agents and that the induced activated phenotypes persist throughout numerous cell doublings and subcultures $(34,35)$.

Mononuclear cell-derived factors may also play a role in moderating or in terminating the fibrotic response as lymphokines and monokines inhibiting the functions of both normal and activated fibroblasts have also been described $(16,23,36-$ 38). We and others have been recently identified those inhibiting fibroblast growth and collagen production as monocyte-derived interferon (IFN) ${ }^{1}-\alpha / \beta$ and T lymphocyte-derived IFN- $\gamma$ (3941). However, in vitro studies of persistent phenotype deactivation have not yet been reported, and to this end, we have attempted to determine whether short-term exposure to IFNs can induce either normal dermal or in vivo-activated scleroderma-derived fibroblasts to persistently express a reducedgrowth and/or a reduced-collagen-producing phenotype.

\section{Methods}

Interferons. Human recombinant DNA-derived (Hu-r) IFN- $\alpha_{2}$ (SCH 30500; sp act, $1.2 \times 10^{8} \mathrm{U} / \mathrm{mg}$ of protein) from Escherichia coli was kindly supplied by Schering Corp., Kenilworth, NJ. Human naturally derived (Hu-n) IFN- $\beta\left(1.4 \times 10^{6} \mathrm{U} / \mathrm{mg}\right)$ was obtained from Lee Biomolecular, Inc., San Diego CA. Hu-r Escherichia coli-derived IFN- $\gamma(1.3$ $\times 10^{7} \mathrm{U} / \mathrm{mg}$ ) was a gift from Genentech, Inc., South San Francisco, CA. The titer of these IFN preparations ranged from $6.6 \times 10^{7}$ to $5 \times 10^{4}$

1. Abbreviations used in this paper: ATCC, American Type Culture Collection; DME, Dulbecco's modified Eagle's medium; Hu-n, human naturally derived; Hu-r, human recombinant DNA-derived; IFN, interferon. 
$\mathrm{U} / \mathrm{ml}$, and after dilution they were added directly to fibroblast cultures to yield a final concentration of $1,000 \mathrm{U} / \mathrm{ml}$.

Fibroblast cultures. Primary fibroblast cultures were initiated from full-thickness 4-mm punch biopsies using the explant method. The explants were cultured in 2-cm ${ }^{2}$ wells (Linbro, Flow Laboratories, Inc., McLean, VA) in exdotoxin-free Dulbecco's modified Eagle's medium (DME) containing $25 \mathrm{mM}$ Hepes, $2 \mathrm{mM}$ glutamine, $100 \mathrm{U} / \mathrm{ml}$ penicillin, and $100 \mu \mathrm{g} / \mathrm{ml}$ streptomycin plus $20 \%$ endotoxin-free fetal calf serum (FCS) (Whittaker-M.A. Bioproducts, Walkersville, MD) at $37^{\circ} \mathrm{C}$ in a $5 \% \mathrm{CO}_{2}$ humidified atmosphere. These primary fibroblast cultures grew to confluency in $\sim 4 \mathrm{wk}$ and were subsequently trypsinized and subcultured in DME-20\% FCS. Nine normal dermal fibroblast lines were established from facial or mammary skin removed from seven women and two men (average age $44.2 \mathrm{yr}$ ) during cosmetic surgery. Three scleroderma fibroblast lines were derived from the clinically involved skin (two forearm, one hip-abdomen junction) of patients with progressive systemic sclerosis (scleroderma) of 1-5 yr duration. Histologic examination of these scleroderma skin specimens showed moderate fibrosis of the papillary and reticular dermis accompanied by perivascular mononuclear cell infiltration, indicating sampled skin was in the active scleroedematous stage of disease. Fibroblast lines derived from these biopsies were chosen for study because a previous investigation indicated that lines developed from active disease areas exhibit appreciably increased production of collagen (42). Two additional scleroderma fibroblast lines, EuKen (CRL 1108 ) and RoDas (CRL 1114), grown from explants of clinically involved scleroderma skin using culture conditions similar to ours (DME $+10 \%$ FCS) were obtained from the American Type Culture Collection (ATCC), Rockville, MD. The characteristics of one of these lines (EuKen) have been previously described (22). The five scleroderma skin donors (four women, one man; average age $45.0 \mathrm{yr}$ ) matched the normal donors for age and sex.

IFN treatment and subculture. Subconfluent fibroblast cultures were set up by placing 10,000 freshly trypsinized fibroblasts, contained in 1 $\mathrm{ml}$ of DME-20\% FCS, into triplicate 2-cm ${ }^{2}$ wells and incubating for 18 $\mathrm{h}$ at $37^{\circ} \mathrm{C}$ in a $5 \% \mathrm{CO}_{2}$ atmosphere to permit adherence to well bottoms. After adherence, medium was removed and replaced with $1 \mathrm{ml}$ of fresh DME-20\% FCS with or without $1,000 \mathrm{U} / \mathrm{ml}$ of either IFN- $\alpha_{2}, \beta$, or $\gamma$, and cultures incubated for an additional $96 \mathrm{~h}$. After $96 \mathrm{~h}$ of growth, fibroblasts were trypsinized, washed, and subcultured at 10,000 cells per $2-\mathrm{cm}^{2}$ well in the absence of IFNs. These subcultures were maintained without media changes and subsequently passaged every $7 \mathrm{~d}$ for an additional 4 wk during which time both untreated normal and untreated scleroderma fibroblasts underwent an average of 18 cell doublings. The growth and collagen production of untreated control and IFN-treated fibroblasts were assessed at each subculture as described below. After the last passage, fibroblasts were again tested for sensitivity to IFN treatment by incubation with $1,000 \mathrm{U} / \mathrm{ml}$ of IFN- $\alpha_{2}, \beta$, or $\gamma$.

Assay of fibroblast growth. Assay of FCS-driven fibroblast growth was performed using asynchronous nonconfluent fibroblast cultures seeded at 10,000 cells per $2-\mathrm{cm}^{2}$ well as described above. Because FCSdriven fibroblast growth is caused by the orchestrated action of the numerous peptide growth factors contained in serum, we performed all the studies reported herein using the same lot of FCS in order to eliminate lot-to-lot variation in these peptide growth factors. After $96 \mathrm{~h}$ of culture in DME-20\% FCS with or without IFNs, growth was assessed by counting the number of trypsin-released cells present using a hemocytometer. Assays were done using triplicate cultures and each culture was counted twice and values are expressed as the number of cells per $2-\mathrm{cm}^{2}$ well. Untreated control cultures reached $\sim 80 \%$ confluency after $96 \mathrm{~h}$ of growth.

Assay of fibroblast collagen production. Assay of collagen production during a 24-h period by steady-state, confluent fibroblasts cultured in the presence of $10 \%$ FCS and ascorbic acid was assessed by $\left[{ }^{3} \mathrm{H}\right]$ proline incorporation into collagenous protein. The use of confluent cultures eliminates growth-related events; the use of $10 \%$ FCS insures optimal levels of collagen production (43), especially in scleroderma-derived fibroblasts (44) and may partially mimic the in vivo environment to which scleroderma fibroblasts are exposed during the edematous and sclero- edematous stages of the disease, inasmuch as the microvascular endothelial cell damage and platelet activation associated with scleroderma would likely expose in situ scleroderma fibroblasts to the same growth and collagen-production stimulatory factors present in FCS (45). A single lot of FCS was used for all studies in order to eliminate variability owing to factors contained in FCS, such as transforming growth factor- $\beta$, which have collagen-production stimulatory activity $(9,30)$. In order to maximize the differences between normal and scleroderma fibroblast collagen production, we employed a suboptimal feeding schedule similar to those previously described $(20,23,41)$, although other studies show that optimal feeding schedules will also reveal the hypercollagen-producing phenotype of scleroderma fibroblasts (22).

Fibroblasts grown to confluency after $7 \mathrm{~d}$ of culture without media changes were trypsinized and plated in triplicate in 96-well microcultures (Microtest III, Falcon Labware, Oxnard, CA) at a near-confluent density of 25,000 fibroblasts per well in $100 \mu \mathrm{l}$ of DME-20\% FCS and incubated for $48 \mathrm{~h}$ to produce a totally confluent monolayer of fibroblasts. The medium was then removed and replaced with $200 \mu \mathrm{l}$ of DME plus $10 \%$ FCS containing $50 \mu \mathrm{g} / \mathrm{ml}$ ascorbic acid with or without IFNs, and incubated for an additional $48 \mathrm{~h}$. Cultures were labeled with $0.5 \mu \mathrm{Ci}$ of $\left[{ }^{3} \mathrm{H}\right]$ proline ( $31 \mathrm{Ci} / \mathrm{mmol}$; Amersham Corp, Arlington Heights, IL) and $50 \mu \mathrm{g} / \mathrm{ml} \beta$-aminopropionitrile for the final $24 \mathrm{~h}$ of culture. The $\left[{ }^{3} \mathrm{H}\right]$ proline incorporation into pepsin-resistant, salt-precipitated extracellular collagen was then determined as previously described $(37,39$, 46). In our hands this assay yields material that is $95 \%$ collagenase-digestible, and the method isolates $<1 \%$ of noncollagenous proteins biosynthetically labeled with $\left[{ }^{3} \mathrm{H}\right]$ tryptophan (39). Results are expressed as disintegrations per minute of $\left[{ }^{3} \mathrm{H}\right]$ collagen per $10^{3}$ cells with cell numbers determined by counting the number of trypsin-released cells present in a series of identically treated microcultures.

\section{Results}

Growth and collagen production of untreated control fibroblasts. The study was initiated with fibroblast lines in both the second and ninth passages. As shown in Table I, growth rates of both

Table I. Growth of Fibroblast Lines

\begin{tabular}{|c|c|c|}
\hline \multirow[b]{2}{*}{ Line no. } & \multicolumn{2}{|l|}{ Fibroblasts } \\
\hline & Normal & Scleroderma \\
\hline & cells per $2-\mathrm{cm}^{2}$ well & cells per $2-\mathrm{cm}^{2}$ well \\
\hline 1 & $182,000^{*}$ & $98,000^{*}$ \\
\hline 2 & $210,000^{*}$ & $102,000^{\star \delta}$ \\
\hline 3 & $77,000^{*}$ & $84,000^{\ddagger}$ \\
\hline 4 & $95,000^{*}$ & $72,000^{\ddagger}$ \\
\hline 5 & $61,000^{\ddagger}$ & $132,000^{\ddagger}$ \\
\hline 6 & $67,000^{\ddagger}$ & \\
\hline 7 & $106,000^{\ddagger}$ & \\
\hline 8 & $68,000^{\ddagger}$ & \\
\hline 9 & $196,000^{\ddagger}$ & \\
\hline Average \pm SEM & $118,000 \pm 20,000^{\prime}$ & $97,000 \pm 10,000^{1}$ \\
\hline \multicolumn{3}{|c|}{$\begin{array}{l}\text { The growth of asynchronous nonconfluent fibroblasts seeded at } 10,000 \\
\text { per } 2 \text {-cm }{ }^{2} \text { well growing in DME- } 20 \% \text { FCS was assessed by counting } \\
\text { the number of trypsin-released cells present after } 4 \mathrm{~d} \text { of growth }(80 \% \\
\text { confluency). Each value is the mean of duplicate hemocytometer } \\
\text { counts of triplicate cultures with standard deviation being } \sim 10 \% \text {. } \\
\text { * Fibroblast lines in ninth passage. } \\
{ }^{\ddagger} \text { Fibroblast lines in second passage. } \\
\text { \$ Scleroderma line } 1 \text { was EuKen and line } 2 \text { was RoDas. } \\
\text { 'Averages are not statistically different }(P>0.05 \text { by two-tailed } t \text { test) }\end{array}$} \\
\hline
\end{tabular}


normal and scleroderma lines at the initiation of the study without IFN treatment covered a two- to threefold range, but the average growth of the nine untreated normal and five untreated scleroderma lines (both in-house and ATCC-derived) was similar to that of 10,000 seeded cells expanding to $\sim 100,000$ cells over $4 \mathrm{~d}$ of growth as has previously been noted $(19,20,22)$. During the 31-d, five-subculture course of the study, the growth rate of these untreated normal and scleroderma lines remained virtually constant with cells initiated in both the second and ninth passages (see Tables III and IV).

The collagen production of individual normal and scleroderma lines at the initiation of the study also varied over a twoto threefold range as illustrated in Table II. However, both inhouse and ATCC-derived scleroderma lines exhibited an elevated collagen production, and the average collagen production of the five untreated scleroderma lines was $69 \%$ greater than the untreated normal cells, suggesting they possessed an activated collagen producing-phenotype as has previously been demonstrated in other studies (19-23). The collagen production of the untreated normal and scleroderma lines studied remained virtually constant over the $31 \mathrm{~d}$ of the study. with the average scleroderma fibroblast collagen production remaining significantly greater than normal fibroblast production for the entire period, regardless of whether cultures were initiated in the second or ninth passage (see Tables V and VI).

Effect of short-term IFN treatment on fibroblast growth. To determine the effect of short-term IFN treatment on fibroblast growth and collagen production, fibroblasts were treated for 4 d with $1,000 \mathrm{U} / \mathrm{ml}$ of IFN- $\alpha_{2}, \beta$, or $\gamma$, and then the growth and collagen production of the fibroblasts subcultured in the absence of IFNs was followed for an additional five passages over $31 \mathrm{~d}$ as described in the Methods.

The mean effect of IFN treatment on the growth of nine normal fibroblast lines is shown in Table III. On day 0 , after 4

Table II. Collagen Production of Fibroblast Lines

\begin{tabular}{lll}
\hline & \multicolumn{2}{l}{$\left[{ }^{3} \mathrm{H}\right]$ Collagen } \\
\cline { 2 - 3 } Line no. & Normal fibroblasts & Scleroderma fibroblasts \\
\hline & $d p m / 10^{3}$ cells & $d p m / 10^{3}$ cells \\
1 & $61^{*}$ & $148^{\star \S}$ \\
2 & $98^{*}$ & $245^{\star}$ \\
3 & $185^{*}$ & $136^{\ddagger}$ \\
4 & $200^{*}$ & $141^{\ddagger}$ \\
5 & $147^{\ddagger}$ & $237^{\ddagger}$ \\
6 & $42^{\ddagger}$ & \\
7 & $74^{\ddagger}$ & \\
8 & $128^{\ddagger}$ & \\
9 & $69^{\ddagger}$ & $181 \pm 24^{\top}$ \\
Average \pm SEM & $112 \pm 18^{\top}$ &
\end{tabular}

The collagen produced during a 24-h period by steady-state, confluent, fibroblasts cultured in DME-10\% FCS-ascorbate was assessed by $\left[{ }^{3} \mathrm{H}\right]$ proline incorporation into pepsin-resistant, salt-precipitated collagen as detailed in Methods. Each value is the mean of triplicate determinations with standard deviation being $\sim 10 \%$.

* Fibroblast lines in ninth passage.

${ }^{\ddagger}$ Fibroblast lines in second passage.

${ }^{8}$ Scleroderma line 1 was EuKen and line 2 was RoDas.

'Averages are statistically different $(P<0.05$ by two-tailed $t$ test).
Table III. Normal Fibroblast Growth after IFN Treatment

\begin{tabular}{|c|c|c|c|c|c|}
\hline \multirow{2}{*}{$\begin{array}{l}\text { Time } \\
\text { posttreatment }\end{array}$} & \multirow{2}{*}{$\begin{array}{l}\text { Subculture } \\
\text { posttreatment }\end{array}$} & \multicolumn{4}{|c|}{ Number of cells } \\
\hline & & Control & Hu-rIFN- $\alpha_{2}$ & Hu-nIFN- $\beta$ & Hu-rIFN- $\gamma$ \\
\hline$d$ & & \multicolumn{4}{|c|}{$10^{3}$ per $2-\mathrm{cm}^{2}$ well $\pm S E M$} \\
\hline 0 & & $118 \pm 20$ & $59 \pm 12^{*}$ & $48 \pm 10^{*}$ & $60 \pm 12^{*}$ \\
\hline 4 & 1 & $110 \pm 18$ & $52 \pm 13^{*}$ & $48 \pm 19^{*}$ & $57 \pm 10^{*}$ \\
\hline 10 & 2 & $107 \pm 25$ & $79 \pm 18$ & $76 \pm 20$ & $70 \pm 13$ \\
\hline 17 & 3 & $89 \pm 18$ & $85 \pm 17$ & $90 \pm 8$ & $83 \pm 16$ \\
\hline 24 & 4 & $107 \pm 3$ & $100 \pm 12$ & $105 \pm 10$ & $109 \pm 15$ \\
\hline 31 & 5 & $116 \pm 17$ & $120 \pm 9$ & $118 \pm 9$ & $123 \pm 8$ \\
\hline
\end{tabular}

Subconfluent cultures of nine normal dermal fibroblast lines were treated with IFNs $(1,000 \mathrm{U} / \mathrm{ml})$ for $96 \mathrm{~h}$ and then the growth of the fibroblasts subcultured in the absence of IFNs was followed for an additional five passages ( 18 cells doublings) over $31 \mathrm{~d}$.

* Values are statistically different from untreated controls $(P<0.05$ by two-tailed $t$ test).

d of treatment with $1,000 \mathrm{U} / \mathrm{ml}$ of either rIFN- $\alpha_{2}$, nIFN- $\beta$, or rIFN- $\gamma$, cell growth was inhibited by $50-60 \%$ as we and others have previously reported $(39,47)$. However, after three subcultures ( $\sim 10$ cell doublings) in the absence of IFNs, cell growth returned to untreated control levels.

The growth of the five scleroderma fibroblast lines was similarly inhibited by $4 \mathrm{~d}$ of treatment with all IFNs as shown in Table IV. Similar to normal fibroblasts, the IFN inhibition of scleroderma fibroblast growth was also a transient effect, with all growth returning to untreated control levels after only three subcultures in the absence of IFNs.

Effect of short-term IFN treatment on fibroblast collagen production. The effect of IFN treatment on the collagen production of normal fibroblasts, shown in Table $\mathrm{V}$, illustrates that on day 0 after $4 \mathrm{~d}$ of treatment, all three IFNs caused collagen production to be inhibited by $50-60 \%$ as we have previously reported (39). Once again, however, the IFN inhibition was only a transient effect, because collagen production returned to untreated control levels after two to three subcultures without IFNs.

In contrast, $4 \mathrm{~d}$ of treatment with all three IFNs not only initially inhibited the collagen production of scleroderma fibro-

Table IV. Scleroderma Fibroblast Growth after IFN Treatment

\begin{tabular}{llcccc}
\hline \multirow{5}{*}{$\begin{array}{l}\text { Time } \\
\text { posttreatment }\end{array}$} & $\begin{array}{l}\text { Subculture } \\
\text { posttreatment }\end{array}$ & \multicolumn{5}{c}{ Number of cells } \\
\cline { 3 - 6 } & Control & Hu-rIFN- $\alpha_{2}$ & Hu-nIFN- $\beta$ & Hu-rIFN- $\gamma$ \\
\hline$d$ & $10^{3}$ per $2-c m^{2}$ well $\pm S E M$ \\
0 & 1 & $97 \pm 10$ & $41 \pm 10^{*}$ & $45 \pm 12^{*}$ & $50 \pm 6^{*}$ \\
4 & 1 & $100 \pm 14$ & $46 \pm 13^{*}$ & $42 \pm 18^{*}$ & $51 \pm 13^{*}$ \\
10 & 2 & $96 \pm 6$ & $69 \pm 10^{*}$ & $58 \pm 25$ & $54 \pm 19$ \\
17 & 3 & $97 \pm 14$ & $95 \pm 5$ & $86 \pm 14$ & $92 \pm 17$ \\
24 & 4 & $105 \pm 21$ & $102 \pm 7$ & $100 \pm 5$ & $90 \pm 8$ \\
31 & 5 & $94 \pm 19$ & $96 \pm 13$ & $95 \pm 15$ & $96 \pm 4$ \\
\hline
\end{tabular}

Subconfluent cultures of five scleroderma fibroblast lines were treated with IFNs $(1,000 \mathrm{U} / \mathrm{ml})$ for $96 \mathrm{~h}$ and then the growth of the fibroblasts subcultured in the absence of IFNs was followed for an additional five passages ( 18 cell doublings) over $31 \mathrm{~d}$.

* Values are statistically different from untreated controls $(P<0.05$ by two-tailed $t$ test). 
Table V. Normal Fibroblast Collagen

Production after IFN Treatment

\begin{tabular}{|c|c|c|c|c|c|}
\hline \multirow{2}{*}{$\begin{array}{l}\text { Time } \\
\text { posttreatment }\end{array}$} & \multirow{2}{*}{$\begin{array}{l}\text { Subculture } \\
\text { posttreatment }\end{array}$} & \multicolumn{4}{|c|}{$\left[{ }^{3} \mathrm{H}\right]$ Collagen } \\
\hline & & Control & Hu-rIFN- $\alpha_{2}$ & Hu-nIFN- $\beta$ & Hu-rIFN- $\gamma$ \\
\hline$d$ & & \multicolumn{4}{|c|}{$\mathrm{dpm} / 10^{3}$ cells $\pm S E M$} \\
\hline 0 & & $112 \pm 18$ & $60 \pm 10^{*}$ & $48 \pm 12^{*}$ & $43 \pm 11^{*}$ \\
\hline 4 & 1 & $106 \pm 16$ & $59 \pm 11^{*}$ & $49 \pm 15^{*}$ & $42 \pm 15^{*}$ \\
\hline 10 & 2 & $100 \pm 15$ & $90 \pm 13$ & $78 \pm 16$ & $86 \pm 16$ \\
\hline 17 & 3 & $96 \pm 11$ & $89 \pm 15$ & $91 \pm 13$ & $91 \pm 5$ \\
\hline 24 & 4 & $109 \pm 7$ & $96 \pm 6$ & $100 \pm 16$ & $105 \pm 17$ \\
\hline 31 & 5 & $112 \pm 12$ & $107 \pm 16$ & $108 \pm 15$ & $102 \pm 18$ \\
\hline
\end{tabular}

Subconfluent cultures of nine normal fibroblast lines were treated with IFNs $(1,000 \mathrm{U} / \mathrm{ml})$ for $96 \mathrm{~h}$ and then the collagen production of the fibroblasts subcultured in the absence of IFNs was followed for an additional five passages ( 18 cell doublings) over $31 \mathrm{~d}$.

* Values are statistically different from untreated controls $(P<0.05$ by two-tailed $t$ test).

blast on day 0 by $50-60 \%$ but the collagen production of these cells, although rising somewhat through the first two passages, remained markedly reduced throughout the entire 31-d, 18-cell division, subculture period in the absence of IFNs (Table VI). Moreover, the reduced amount of collagen produced by these inhibited cells was now approximately equal to that produced by untreated normal fibroblasts. These data suggest IFNs have persistently reduced the collagen-producing phenotype of these scleroderma-derived fibroblasts.

Response of 31-d posttreatment fibroblasts to reexposure to IFNs. To determine whether these reduced-phenotype fibroblasts now behave as normal fibroblasts by responding to IFNs with a transient further reduction of collagen production, we reexposed 31-d posttreatment fibroblasts to the IFNs with which they had originally been treated. However, scleroderma fibroblast collagen production was now largely unresponsive to IFN treatment and was not markedly inhibited (Table VII). This was in contrast to the significant reinhibition of the growth of scleroderma fibro-

Table VI. Scleroderma Fibroblast Collagen Production after IFN Treatment

\begin{tabular}{|c|c|c|c|c|c|}
\hline \multirow{2}{*}{$\begin{array}{l}\text { Time } \\
\text { posttreatment }\end{array}$} & \multirow{2}{*}{$\begin{array}{l}\text { Subculture } \\
\text { posttreatment }\end{array}$} & \multicolumn{4}{|c|}{$\left[{ }^{3} \mathrm{H}\right]$ Collagen } \\
\hline & & Control & Hu-rIFN- $\alpha_{2}$ & Hu-nIFN- $\beta$ & Hu-rIFN- $\gamma$ \\
\hline$d$ & & \multicolumn{4}{|c|}{$\mathrm{dpm} / 10^{3}$ cells $\pm S E M$} \\
\hline 0 & & $181 \pm 24$ & $82 \pm 13$ & $74 \pm 14$ & $56 \pm 9$ \\
\hline 4 & 1 & $166 \pm 11$ & $81 \pm 7$ & $72 \pm 13$ & $50 \pm 9$ \\
\hline 10 & 2 & $174 \pm 13$ & $106 \pm 8$ & $98 \pm 7$ & $101 \pm 8$ \\
\hline 17 & 3 & $169 \pm 20$ & $104 \pm 14$ & $103 \pm 15$ & $108 \pm 11$ \\
\hline 24 & 4 & $160 \pm 19$ & $104 \pm 16$ & $101 \pm 16$ & $98 \pm 13$ \\
\hline 31 & 5 & $155 \pm 14$ & $105 \pm 14$ & $95 \pm 13$ & $85 \pm 11$ \\
\hline
\end{tabular}

Subconfluent cultures of five scleroderma fibroblast lines were treated with IFNs $(1,000 \mathrm{U} / \mathrm{ml})$ for $96 \mathrm{~h}$ and then the collagen production of the fibroblasts subcultured in the absence of IFNs was followed for an additional five passages ( 18 cell doublings) over $31 \mathrm{~d}$. All IFN treatment values are statistically different from untreated controls $(P<0.05$ by two-tailed $t$ test).
Table VII. Response of 31-d Posttreatment

Fibroblasts to Reexposure to IFNs

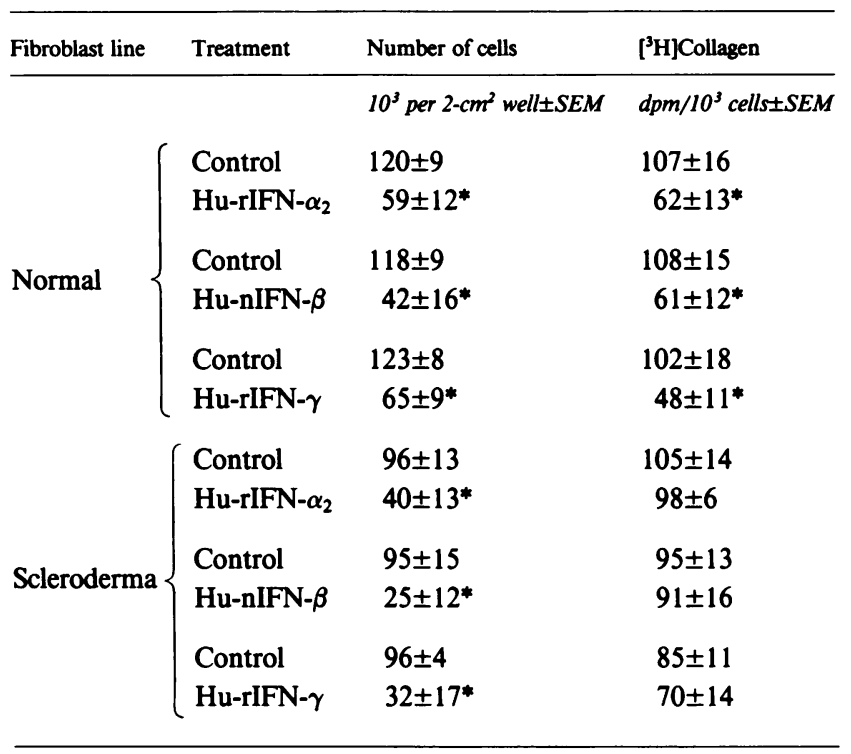

Subconfluent cultures of nine normal and five scleroderma fibroblast lines were treated with IFNs $(1,000 \mathrm{U} / \mathrm{ml})$ for $96 \mathrm{~h}$ and then subcultured in the absence of IFNs for five passages over $28 \mathrm{~d}$. Cultures of these fibroblasts, set up as described in Methods for assays of fibroblast growth or collagen production, were assayed for growth or collagen production on day 31 after incubation in the absence or presence of $1,000 \mathrm{U} / \mathrm{ml}$ of the IFNs to which they had originally been exposed.

* Values are statistically different from controls not reexposed to IFNs $(P<0.05$ by two-tailed $t$ test).

blasts and the growth and collagen production of normal fibroblasts. Thus, although IFN-treated scleroderma fibroblasts produce normal amounts of collagen, they do not seem to possess all the characteristics of normal fibroblasts inasmuch as their collagen production could not be reinhibited by subsequent IFN treatment.

\section{Discussion}

Because we had recently identified IFN- $\gamma$ as the lymphokine and IFN- $\alpha$ and $\beta$ as the monokines responsible for inhibiting fibroblast growth and collagen production, in this report we investigated whether IFNs could persistently normalize the activity of cultured fibroblast previously activated in vivo. We chose fibroblasts derived from scleroderma skin as our activated model because they display persistently activated phenotypes characterized by the hyperproduction of collagens, fibronectin, and glycosaminoglycans but normal growth and collagenase production rates (19-24). In this way we could study the effect of IFNs on both activated (collagen production) and normal (growth) functions in the same fibroblast line. Moreover, similar transcription-regulatory mechanisms may be responsible for the increased collagen production of scleroderma fibroblasts and the decreased collagen production of IFN-treated fibroblasts, inasmuch as scleroderma fibroblasts have increased levels of procollagen messenger RNA (mRNA), and IFN treatment decreases the procollagen mRNA levels of both scleroderma and normal fibroblasts $(41,48,49)$. 
The results of our study demonstrate that both scleroderma and normal fibroblasts are sensitive to the initial inhibitory effects of either IFN- $\alpha, \beta$, or $\gamma$, inasmuch as the growth and collagen production of both fibroblast types were inhibited $40-60 \%$ by $96 \mathrm{~h}$ of IFN treatment. However, those fibroblast functions that were normally active, namely, the growth and collagen production of normal fibroblasts and the growth of scleroderma fibroblasts, returned to untreated levels during subsequent subculture in the absence of IFNs. In contrast, scleroderma fibroblast collagen production, an activated function, remained inhibited for 18 cell doublings in the absence of IFNs. This population of deactivated scleroderma fibroblasts, although producing normal amounts of collagen, was however, not identical to the normal unactivated fibroblast populations studied because its collagen production was no longer sensitive to IFN-mediated inhibition. These data suggest, that although IFNs are only transient inhibitors of normal fibroblast functions, they do indeed act as persistent deactivators of at least one activated fibroblast function. Whether IFNs inhibit other activated fibroblast functions such as glycosaminoglycan or fibronectin production in a persistent manner remains to be investigated. However, IFN- $\gamma$ has been reported to inhibit both the collagen and fibronectin production of rheumatoid synovial fibroblast-like cells, although persistence of inhibition was not investigated (50).

In the present study, we have not attempted to analyze the types of collagens produced before and after IFN treatment nor the mechanism by which IFNs inhibit collagen production. However, previous reports demonstrate that in the presence of FCS both normal and scleroderma fibroblasts produce type I and III collagen in a ratio of $\sim 5: 1(22)$, and short-term IFN treatment causes a coordinate decrease in the production of both type I and III collagen in normal and scleroderma fibroblasts as assessed by procollagen and procollagen mRNA levels $(40,41$, 49). The IFN-induced decreases in procollagen mRNAs were proportionate to procollagen reduction and no detectable increase of collagen degradation products was observed, suggesting that IFNs act at a transcriptional level $(40,41,49)$. Whether IFNs inhibit only the procollagen mRNA synthesis induced by the transforming growth factor- $\beta$ contained in FCS-supplemented media $(9,39)$, or also inhibit basal level procollagen mRNA synthesis was not assessed. However, we have previously shown that IFNs or mononuclear cell supernatants containing IFNs can inhibit both normal and scleroderma fibroblast collagen production in cultures containing low $(1 \%)$ or no FCS $(37,39$, unpublished observations).

The mechanism responsible for the observed emergence of a persistently reduced collagen-producing phenotype after transient exposure of scleroderma fibroblasts to IFNs may involve either clonal selection or phenotype differentiation. Because the activated scleroderma fibroblasts that we studied were growing when treated with IFNs, it is possible that the deactivation of collagen production that we observed was due to clonal selection inasmuch as the IFNs were also growth inhibitors. Moreover, the collagen production of the deactivated scleroderma fibroblast was insensitive to subsequent IFN exposure, suggesting that a subpopulation of IFN-sensitive, high collagen-producing cells had been eliminated. Alternatively, growth-independent phenotypic differentiation with concurrent loss of sensitivity of collagen production to IFN treatment may have occurred because IFNs clearly inhibit collagen production in static, nongrowing cultures of both normal and scleroderma fibroblasts $(39-41,49)$. Such a theory is supported by our preliminary studies, which demonstrate that when confluent, nongrowing cultures of two lines of scleroderma fibroblasts are transiently treated with IFNs and then maintained for $4 \mathrm{wk}$ as confluent static cultures in $0.5 \%$ FCS $(51)$, their collagen production is both persistently inhibited and insensitive to subsequent IFN treatment. Thus it is likely that alterations in cell growth are not required for the IFN-induced phenotype changes we have observed.

Having demonstrated that IFNs deactivate scleroderma fibroblasts it is logical for us to suggest that the excessive fibrosis seen in vivo in scleroderma may be due to the deficient in situ production of IFNs by infiltrating monocytes/macrophages and $T$ lymphocytes during the early edematous and scleroedematous stages of disease (26-29). This, however, may not be the case, because peripheral blood mononuclear cells from scleroderma patients produce normal amounts of collagen production-inhibitory factors now known to be IFNs $(23,37)$. Production of excessive amounts of a collagen production-stimulatory lymphokine by scleroderma peripheral blood mononuclear cells has, however, been reported (52), and this factor may be responsible for in vivo activation of hypercollagen-producing fibroblasts. Nevertheless, our results suggest that scleroderma may be amenable to therapy with IFNs or IFN inducers in that increasing in vivo levels of IFN would likely block the effect of excessive stimulatory factors, resulting in fibroblast deactivation. Similarly, other diseases characterized by pathological fibrosis, such as forms of pulmonary and liver fibrosis, chronic graft-vs.-host disease, rheumatoid arthritis, localized scleroderma, keloids, and hypertrophic scars, may also benefit from IFN treatment, regardless of the mechanisms leading to aberrant fibroblast activation. This concept of general in vivo fibroblast deactivation by IFNs is supported by a recently reported study showing that in vivo administration of IFN- $\gamma$ prevents accumulation of collagen in the lungs of bleomycin-treated mice (53) and by reports of delayed wound healing in viral infected mice that may be due to the presence of high levels of virally induced IFN (54).

\section{Acknowledgments}

We wish to thank Genentech, Inc. and the Schering Corp. for the gift of recombinant DNA-derived IFN- $\gamma$ and $\alpha$, respectively.

This work was supported by grants from the Lester I. Conrad Research Foundation and the Veterans Administration Research Service.

\section{References}

1. Bullough, W. S., and E. B. Laurence. 1960. The control of mitotic activity in mouse skin: dermis and hypodermis. Exp. Cell. Res. 21:394405.

2. Wiestner, M., T. Krieg, D. Horlein, R. W. Glanville, P. Fietzek, and P. K. Muller. 1979. Inhibitory effect of procollagen peptides on collagen biosynthesis in fibroblast cultures. J. Biol. Chem. 254:70167023.

3. Clark, R. A. F. 1985. Cutaneous tissue repair: basic biological considerations. J. Am. Acad. Dermatol. 13:701-725.

4. Korotzer, T. I., J. A. Clagett, W. P. Kolb, and R. C. Page. 1980. Complement-dependent induction of DNA synthesis and proliferation of human diploid fibroblast. J. Cell. Physiol. 105:503-512.

5. Pohjanpelto, P. 1978. Stimulation of DNA synthesis in human fibroblasts by thrombin. J. Cell. Physiol. 95:189-194.

6. Deuel, T. F., and J. S. Huang. 1984. Platelet-derived growth factor: structure, function and roles in normal and transformed cells. J. Clin. Invest. 74:669-676.

7. Castor, C. W., J. W. Miller, and D. A. Walz. 1983. Structural and 
biological characteristics of connective tissue activating peptide (CTAPIII), a major human platelet-derived growth factor. Proc. Natl. Acad. Sci. USA. 80:765-769.

8. Oka, Y., and D. N. Orth. 1983. Human plasma epidermal growth factor/beta-urogastrone is associated with blood platelets. J. Clin. Invest. 72:249-259.

9. Ignotz, R. A., and J. Massague. 1986. Transforming growth factorbeta stimulates the expression of fibronectin and collagen and their incorporation into extracellular matrix. J. Biol. Chem. 261:4337-4345.

10. Clemmons, D. R., and J. J. Van Wyk. 1981. Somatomedin-C and platelet-derived growth factor stimulate human fibroblast replication. J. Cell. Physiol. 106:361-367.

11. Myers, S. L., and C. W. Castor. 1980. Connective tissue activation. $\mathrm{XV}$. Stimulation of glycosaminoglycan and DNA synthesis by a polymorphonuclear leucocyte factor. Arthritis Rheum. 23:556-563.

12. Schmidt, J. A., S. B. Mizel, D. Cohen, and I. Green. 1982. Interleukin 1: a potential regulator of fibroblast proliferation. J. Immunol. 128:2177-2182.

13. Krane, S. M., J. M. Dayer, L. S. Simon, and M. S. Byrne. 1985. Mononuclear cell-conditioned medium containing mononuclear cell factor (MCF), homologous with interleukin 1, stimulates collagen and fibronectin synthesis by adherent rheumatoid synovial cells: effects of prostaglandin $\mathrm{E}_{2}$ and indomethacin. Collagen Relat. Res. 5:99-117.

14. Dayer, J. M., B. deRochemonteix, B. Burrus, S. Demczuk, and C. A. Dinarello. 1986. Human recombinant interleukin 1 stimulates collagenase and prostaglandin $\mathrm{E}_{2}$ production by human synovial cells. J. Clin. Invest. 77:645-648.

15. Wahl, S. M., and C. L. Gately. 1982. Modulation of fibroblast growth by a lymphokine of human $\mathrm{T}$ cell and continuous $\mathrm{T}$ cell line origin. J. Immunol. 130:1226-1230.

16. Postlethwaite, A. E., G. N. Smith, C. L. Mainardi, J. M. Seyer, and A. H. Kang. 1984. Lymphocyte modulation of fibroblast function in vitro: stimulation and inhibition of collagen production by different effector molecules. J. Immunol. 132:2470-2477.

17. Castor, C. W. 1971. Abnormalities of connective tissue cells cultured from patients with rheumatoid arthritis: defective regulation of hyaluronate and collagen formation. J. Lab. Clin. Med. 77:65-75.

18. Abergel, P. R., D. Pizzurro, C. A. Meeker, G. Lask, L. Y. Matsuoka, R. R. Minor, M. L. Chu, and J. Uitto. 1985. Biochemical composition of the connective tissue in keloids and analysis of collagen metabolism in keloid fibroblast cultures. J. Invest. Dermatol. 84:384-390.

19. LeRoy, E. C. 1974. Increased collagen synthesis by scleroderma skin fibroblasts in vitro. J. Clin. Invest. 54:880-889.

20. Buckingham, R. B., R. K. Prince, G. P. Rodnan, and F. Taylor 1978. Increased collagen accumulation in dermal fibroblast cultures from patients with progressive systemic sclerosis (scleroderma). J. Lab. Clin. Med. 92:5-21.

21. Fleischmajer, R., J. S. Perlish, T. Krieg, and R. Timpl. 1981. Variability in collagen and fibronectin synthesis by scleroderma fibroblast in primary culture. J. Invest. Dermatol. 76:400-403.

22. Uitto, J., E. A. Bauer, and A. Z. Eisen. 1979. Scleroderma: increased biosynthesis of triple-helical type I and type III procollagens associated with unaltered expression of collagenase by skin fibroblast in culture. J. Clin. Invest. 64:921-930.

23. Jimenez, S. A., W. M. McArthur, R. I. Bashey, and J. Rosenbloom. 1985. Selective inhibition of excessive scleroderma fibroblast collagen production by lymphokines from normal Human mononuclear cells. Arthritis Rheum. 28:502-510

24. Buckingham, R. B., R. K. Prince, and G. P. Rodnan. 1983. Progressive systemic sclerosis (PSS, scleroderma) dermal fibroblasts synthesize increased amounts of glycosaminoglycan. J. Lab. Clin. Med. 101:659669.

25. Dayer, J. M., S. M. Krane, R. G. G. Russel, and D. R. Robinson. 1976. Production of collagenase and prostaglandins by isolated adherent, rheumatoid synovial cells. Proc. Natl. Acad. Sci. USA. 73:945-949.

26. Wahl, S. M., D. G. Malone, and R. L. Wilder. 1985. Spontaneous production of fibroblast-activating factor(s) by synovial inflammatory cells. J. Exp. Med. 161:210-222.

27. Fleischmajer, R., J. S. Perlish, and W. P. West. 1977. Ultrastructure of cutaneous cellular infiltrates in scleroderma. Arch. Dermatol. 113:1661-1666.

28. Roumm, A. D., T. L. Whiteside, T. A. Medsger, and G. P. Rodnan. 1984. Lymphocytes in the skin of patients with progressive systemic sclerosis. Arthritis Rheum. 27:645-653.

29. Whiteside, T. L., J. G. Worrall, R. K. Prince, R. B. Buckingham, and G. P. Rodnan. 1985. Soluble mediators from mononuclear cells increase the synthesis of glycosaminoglycan by dermal fibroblast cultures derived from normal subjects and progressive systemic sclerosis patients. Arthritis Rheum. 28:188-197.

30. Sporn, M. B., A. B. Roberts, L. M. Wakefield, and R. K. Assoian. 1986. Transforming growth factor-beta: biological function and chemical structure. Science (Wash. DC). 233:532-534.

31. Bitterman, P. B., M. D. Wewers, S. I. Rennard, S. Adelberg, and R. G. Crystal. 1986. Modulation of alveolar macrophage-driven fibroblast proliferation by alternate macrophage mediators. J. Clin. Invest. 77:700708.

32. Beutler, B., and A. Cerami. 1986. Cachectin and tumor necrosis factor as two sides of the same biological coin. Nature (Lond.). 320:584589.

33. Mornex, J.-F., Y. Martinet, K. Yamauchi, P. B. Bitterman G. R. Grotendorst, A. Chytil-Weir, G. R. Martin, and G. Crystal. 1986. Spontaneous expression of the c-sis gene and release of platelet-derived growth factorlike molecule by human alveolar macrophages. J. Clin. Invest. 78:61-66.

34. Worrall, J. G., T. L. Whiteside, R. K. Prince, R. B. Buckingham I. Stachura, and G. P. Rodnan. 1986. Persistence of scleroderma-like phenotype in normal dermal fibroblasts after prolonged exposure to soluble mediators from mononuclear cells. Arthritis Rheum. 29:54-64.

35. Korn, J. H. 1983. Fibroblast prostaglandin $E_{2}$ synthesis: persistence of an abnormal phenotype after short-term exposure to mononuclear cell products. J. Clin. Invest. 71:1240-1246.

36. Korn, J. H., P. V. Halushka, and E. C. Leroy. 1980. Mononuclear cell modulation of connective tissue function: suppression of fibroblast growth by stimulation of endogenous prostaglandin production. J. Clin. Invest. 65:543-554.

37. Duncan, M. R., J. S. Perlish, and R. Fleischmajer. 1984. Lymphokine/monokine inhibition of fibroblast proliferation and collagen production: role in progressive systemic sclerosis (PSS). J. Invest. Dermatol. 83:377-384.

38. Jimenez, S. A., W. A. McArthur, and J. Rosenbloom. 1979. Inhibition of collagen synthesis by mononuclear cell supernates. J. Exp. Med. 150:1421-1431.

39. Duncan, M. R., and B. Berman. 1985. Gamma-interferon is the lymphokine and beta-interferon the monokine responsible for inhibition of fibroblast collagen production and late but not early fibroblast proliferation. J. Exp. Med. 162:516-527.

40. Jimenez, S. A., B. Freundlich, and J. Rosenbloom. 1984. Selective inhibition of diploid fibroblast collagen synthesis by interferons. J. Clin. Invest. 74:1112-1116.

41. Rosenbloom, J., G. Feldman, B. Freundlich, and S. A. Jimenez. 1986. Inhibition of excessive scleroderma fibroblast collagen synthesis by recombinant gamma interferon. Arthritis Rheum. 29:851-856.

42. Vuorio, T. K., V.-L. Kahari, A. Lehtonen, and E. I. Vuorio. 1984. Fibroblast activation in scleroderma. Scand. J. Rheumatol. 13: 229-237.

43. Booth, B. A., K. L. Polak, and J. Uitto. 1980. Collagen biosynthesis by human fibroblasts: optimization of the culture conditions for synthesis of type I and type III procollagens. Biochim. Biophys. Acta. 607:145160.

44. Bashey, R. I., and S. A. Jimenez. 1977. Increased sensitivity of scleroderma fibroblasts in culture to stimulation of protein and collagen synthesis by serum. Biochim. Biophys. Acta. 76:1214-1222. 
45. Kahaleh, M. B., I. Osborn, and E. C. LeRoy. 1982. Elevated levels of circulating platelet aggregates and beta-thromboglobulin in scleroderma. Ann. Intern. Med. 96:610-613.

46. Webster, D. F., and W. Harvey. 1979. A quantitative assay for collagen synthesis in microwell fibroblast cultures. Anal. Biochem. 96: 220-224.

47. Pfeffer, L. M., J. S. Murphy, and I. Tamm. 1979. Interferon effects on the growth and division of human fibroblasts. Exp. Cell Res. 121:111-120.

48. Graves, P. N., I. K. Weiss, J. S. Perlish, and R. Fleischmajer. 1983. Increased procollagen mRNA levels in scleroderma skin fibroblasts. J. Invest. Dermatol. 80:130-132.

49. Rosenbloom, J., G. Feldman, B. Freundlich, and S. A. Jimenez. 1984. Transcriptional control of human diploid fibroblast collagen syn- thesis by gamma-interferon. Biochem. Biophys. Res. Commun. 123:365372.

50. Amento, E. P., A. K. Bhan, K. G. McCullagh, and S. M. Krane. 1985. Influences of gamma interferon on synovial fibroblast-like cells: Ia induction and inhibition of collagen synthesis. J. Clin. Invest. 76:837848

51. Dell'Orco, R. T. 1974. Maintenance of human diploid fibroblasts as arrested populations. Fed. Proc. 33:1969-1972.

52. Cathart, M. K., and R. S. Krakauer. 1981. Immunological enhancement of collagen accumulation in progressive systemic sclerosis (PSS). Clin. Immunol. Immunopathol. 21:128-133.

53. Giri, S. N., D. M. Hyde, and B. J. Marafino, Jr. 1986. Ameliorating effect of murine interferon gamma on bleomycin-induced lung collagen fibrosis in mice. Biochem. Med. Metab. Biol. 36:194-197.

54. Kenyon, A. J. 1983. Delayed wound healing in mice associated with viral alteration of macrophages. Am. J. Vet. Res. 44:652-656. 